University of Texas at El Paso

ScholarWorks@UTEP

$1-2017$

\title{
Why Decimal System and Binary System Are the Most Widely Used: A Possible Explanation
}

Gerardo Muela

The University of Texas at El Paso, gdmuela@miners.utep.edu

Follow this and additional works at: https://scholarworks.utep.edu/cs_techrep

Part of the Computer Sciences Commons

Comments:

Technical Report: UTEP-CS-17-04

\section{Recommended Citation}

Muela, Gerardo, "Why Decimal System and Binary System Are the Most Widely Used: A Possible Explanation" (2017). Departmental Technical Reports (CS). 1114.

https://scholarworks.utep.edu/cs_techrep/1114

This Article is brought to you for free and open access by the Computer Science at ScholarWorks@UTEP. It has been accepted for inclusion in Departmental Technical Reports (CS) by an authorized administrator of ScholarWorks@UTEP.For more information, please contact Iweber@utep.edu. 


\title{
Why Decimal System and Binary System Are the Most Widely Used: A Possible Explanation
}

\author{
Gerardo Muela \\ Department of Computer Science \\ University of Texas at El Paso \\ El Paso, TX 79968, USA \\ gdmuela@miners.utep.edu
}

\begin{abstract}
What is so special about numbers 10 and 2 that decimal and binary systems are the most widely used? One interesting fact about 10 is that when we start with a unit interval and we want to construct an interval of half width, then this width is exactly $5 / 10$; when we want to find a square of half area, its sides are almost exactly $7 / 10$, and when we want to construct a cube of half volume its sides are almost exactly $8 / 10$. In this paper, we show that 2,4 , and 10 are the only numbers with this property - at least among the first billion numbers. This may be a possible explanation of why decimal and binary systems are the most widely used.
\end{abstract}

\section{Formulation of the Problem}

Problem. What is so special about numbers 10 and 2 that decimal and binary systems are the most widely used?

This questions was raised, e.g., in [1].

Observation. One interesting fact about 10 is the following:

- When we start with a unit interval and we want to constrict an interval of half width, then this width is exactly $1 / 2=5 / 10$.

- When we start with a unit square and want to find a square of area $1 / 2$, its sides are $\sqrt{1 / 2}$, which is almost exactly $7 / 10$ :

$$
\left|\sqrt{\frac{1}{2}}-\frac{7}{10}\right|<\frac{1}{100} .
$$

- When we start with a unit cube and want to find a cube of volume $1 / 2$, its sides are $\sqrt[3]{1 / 2}$, which is almost exactly $8 / 10$ :

$$
\left|\sqrt[3]{\frac{1}{2}}-\frac{8}{10}\right|<\frac{1}{100}
$$


So, whether we want to construct a piece of land which is (almost) exactly of half-area, or a piece of gold which is (almost) exactly of half-volume, decimal systems is very convenient.

Are there any other numbers with this property? Maybe here are other bases $b$ with this property, i.e., bases $b$ for which, for appropriate numbers $n_{1}$, $n_{2}$, and $n_{3}$, we have

$$
\left|\frac{1}{2}-\frac{n_{1}}{b}\right|<\frac{1}{b^{2}}, \quad\left|\sqrt{\frac{1}{2}}-\frac{n_{2}}{b}\right|<\frac{1}{b^{2}}, \quad\left|\sqrt[3]{\frac{1}{2}}-\frac{n_{3}}{b}\right|<\frac{1}{b^{2}} .
$$

What we do in this paper. In this paper, we show that - at least among the first billion numbers $b$ - only the numbers $b=2, b=4$, and $b=10$ satisfy this property.

Base 4 is, in effect, the same as the binary system - we just group two binary digits to get one 4-ary digit, just like we get an 8-ary system when we group three binary digits or 16-based system when we group 4 binary digits together.

Thus, the above result may be a good explanation of why decimal and binary systems are the most widely used.

\section{Analysis of the Problem}

Considering the first condition. Let us first consider the first of the desired inequalities: $\left|\frac{1}{2}-\frac{n_{1}}{b}\right|<\frac{1}{b^{2}}$. When the base is even, i.e., when $b=2 k$ for some integer $k$, then this property is clearly satisfied: indeed, in this case, for $n_{1}=k$, we get $\frac{n_{1}}{b}=\frac{1}{2}$ and thus, $\left|\frac{1}{2}-\frac{k}{b}\right|=0<\frac{1}{b^{2}}$.

On the other hand, if $b$ is odd, i.e., if $b=2 k+1$ for some natural number $k \geq 1$, then, for $\frac{1}{2}=\frac{k+0.5}{2 k+1}=\frac{k+0.5}{b}$, the closest fractions of the type $\frac{n_{1}}{b}$ are the fractions $\frac{k}{b}$ and $\frac{k+1}{b}$. For both these fractions, we have

$$
\left|\frac{k+0.5}{2 k+1}-\frac{k}{2 k+1}\right|=\left|\frac{k+0.5}{2 k+1}-\frac{k+1}{2 k+1}\right|=\frac{0.5}{2 k+1}=\frac{1}{2 \cdot(2 k+1)}=\frac{1}{2 b} .
$$

The desired inequality thus takes the form $\frac{1}{2 b}<\frac{1}{b^{2}}$, which is equivalent to $2 b>b^{2}$ and $2>b$. However, odd bases start with $b=3$. So, the first condition cannot be satisfied by odd bases $b$.

Thus, the first condition is equivalent to requiring that the base $b$ is an even number.

How do we check the second condition. If we check the second condition $\left|\sqrt{\frac{1}{2}}-\frac{n_{2}}{b}\right|<\frac{1}{b^{2}}$ literally, then we need to consider all possible values $n_{2}$ from 0 
to $b$. However, this can avoided if we multiply both sides of the desired inequality by $b$ and consider the equivalent inequality $\left|b \cdot \sqrt{\frac{1}{2}}-n_{2}\right|<\frac{1}{b}$. In this case, we can easily see that $n_{2}$ is the nearest integer to the product $b \cdot \sqrt{\frac{1}{2}}$ :

$$
n_{2}=\left[b \cdot \sqrt{\frac{1}{2}}\right]
$$

where $[x]$ denotes the nearest integer to the real number $x$. In these terms, the desired inequality takes the form

$$
\left|b \cdot \sqrt{\frac{1}{2}}-\left[b \cdot \sqrt{\frac{1}{2}}\right]\right|<\frac{1}{b}
$$

This is the inequality that we will check.

How to check the third condition. Similarly, if we check the third condition $\left|\sqrt[3]{\frac{1}{2}}-\frac{n_{3}}{b}\right|<\frac{1}{b^{2}}$ literally, then we need to consider all possible values $n_{3}$ from 0 to $b$. However, this can avoided if we multiply both sides of the desired inequality by $b$ and consider the equivalent inequality $\left|b \cdot \sqrt[3]{\frac{1}{2}}-n_{3}\right|<\frac{1}{b}$. In this case, we can easily see that $n_{3}$ is the nearest integer to the product $b \cdot \sqrt[3]{\frac{1}{2}}$ :

$$
n_{3}=\left[b \cdot \sqrt[3]{\frac{1}{2}}\right]
$$

where $[x]$ denotes the nearest integer to the real number $x$. In these terms, the desired inequality takes the form

$$
\left|b \cdot \sqrt[3]{\frac{1}{2}}-\left[b \cdot \sqrt[3]{\frac{1}{2}}\right]\right|<\frac{1}{b}
$$

This is the inequality that we will check.

The checking. For each even number $b$ from 2 to $10^{9}$, we checked whether this number satisfies both conditions (2) and (3). A simple Java program for this checking is given in the appendix.

The result of the checking. The result is that among all the bases $b$ from 1 to $10^{9}$, both roots are only well approximated for $b=2, b=4$, and $b=10$. Thus, only for these three bases, the desired condition (1) is satisfied.

This may explain why decimal and binary systems are the most frequently used. 
Natural conjecture. We have checked all the values $b$ until $10^{9}$. This makes us conjecture that out of all possible natural numbers $b \geq 2$, only the numbers $2,4,10$ satisfy the property (1).

\section{References}

1. D. E. Knuth, The Art of Computer Programming, Addison-Wesley Professional, Boston, Massachusetts, 2011.

\section{A Code}

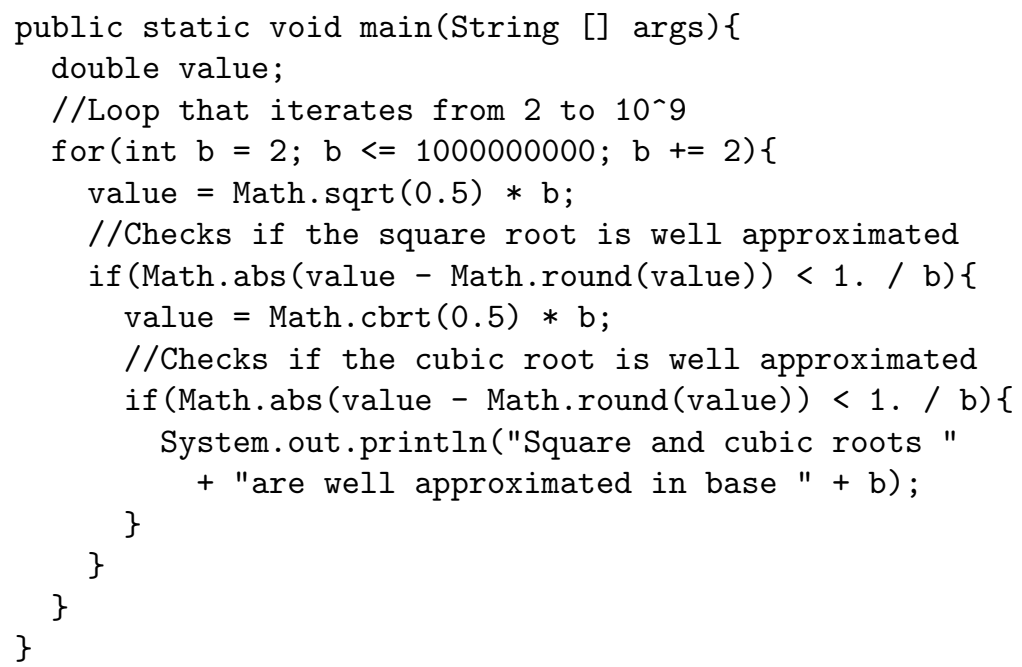

